ORIGINAL ARTICLE

\title{
Validation of fan beam dual energy $x$ ray absorptiometry for body composition assessment in adults aged 18-45 years
}

\author{
J Norcross, M D Van Loan
}

Br J Sports Med 2004;38:472-476. doi: 10.1136/bjsm.2003.005413

See end of article for authors' affiliations .....................

Correspondence to: Dr Van Loan, One Shields Avenue, University of California, Davis, CA 95616, USA; mvanloan@ whnrc.usda.gov

Accepted 21 May 2003
Background: Pencil beam dual energy $x$ ray absorptiometry (DXA) has been shown to provide valid estimates of body fat (\%BF), but DXA fan beam technology has not been adequately tested to determine its validity.

Objective: To compare \%BF estimated from fan beam DXA with \%BF determined using two and three compartment $(2 \mathrm{C}, 3 \mathrm{C})$ models.

Methods: Men $(n=25)$ and women $(n=31)$, aged 18-41 years, participated in the study. Body density, from hydrostatic weighing, was used in the $2 \mathrm{C}$ estimate of \%BF; DXA was used to determine bone mineral content $(B M C)$ for the $3 C$ estimate of \% BF calculated using body density and $B M C\left(3 C_{B M C}\right)$. DXA was also used to determine \%BF. Analysis of variance was used to test for significant differences in \%BF between sexes and among methods.

Results: Women were significantly shorter, weighed less, had less fat free mass, and a higher \%BF than men. No significant differences were found among methods $\left(2 C, 3 C_{B M C}, D X A\right)$ for determination of \% $B F$ in either sex. Although not significant, Bland-Altman plots showed that DXA gave higher values for \%BF than the $2 C$ and $3 C_{B M C}$ methods.

Conclusion: DXA determination of \%BF was not different from that of the $2 \mathrm{C}$ and $3 C_{B M C}$ models in this group of young adults. However, to validate fan beam DXA fully as a method for body composition assessment in a wide range of individuals and populations, comparisons are needed that use a $4 \mathrm{C}$ model with a measure of total body water and BMC.
$\mathrm{T}$ he technique of dual energy $x$ ray absorptiometry (DXA) has been used to measure bone mineral content (BMC), bone mineral density (BMD), and body composition for almost two decades. ${ }^{1-3}$ This technique relies on assumptions that the human body can be subdivided into three compartments: BMC, fat mass (FM), and bone free lean mass (BFLM). If DXA accurately and reliably predicts percentage body fat $(\% \mathrm{BF})$, it could replace more physically demanding techniques such as hydrostatic weighing (HW). The DXA measurement is fast, involves no physical discomfort, and allows people to be fully dressed as long as there is no metal in the clothing or on their person. For these reasons, it is believed that DXA can be a useful tool for measuring body composition in a laboratory, fitness or wellness programme, and in clinical settings.

A considerable amount of methodology related research has been conducted with DXA during the past two decades. Early research focused on the validation of pencil beam DXA measurements with standard laboratory methods including multicompartment models. ${ }^{1-5}$ Although results from these investigations were generally positive, several areas of concern were identified as a result of these investigations. Firstly, Laskey and colleagues ${ }^{6}$ made the observation that the accuracy in determination of body composition varied depending on the anterior-posterior thickness of the subject. Similarly, Jebb and coworkers ${ }^{7}$ found that DXA estimates of body composition were less accurate if the anterior-posterior depth of the subject, when supine on the DXA scan table, was $\geqslant 25 \mathrm{~cm}$. These investigators concluded that a large anteriorposterior tissue mass was responsible for the attenuation of the dual energy $x$ rays, and thus less accurate results were obtained.
Tothill et $\mathrm{al}^{8}$ compared body composition results from DXA instruments developed by three different manufacturers. Equivalent results were not obtained. A possible explanation lies in the software developed for the determination of body composition. Van Loan et al showed that different software versions developed by a single manufacturer for use with a specified instrument produced significantly different estimates of body composition. The observations of Tothill et al ${ }^{8}$ and Van Loan et $a l^{9}$ confirmed the need to use the same instrument and software version during the course of a research investigation to ensure reliable results.

In general, research with pencil beam DXA has been positive. $^{10}{ }^{11}$ However, more recent DXA development has included fan beam technology. This technology includes changes in hardware and considerable software development for the assessment of body composition. The fan beam array distributes the $x$ ray across a wider area, shaped like an open fan. The $x$ rays overlap and require multiple detectors to measure the $x$ ray signal. Different manufacturers have different arrangements for the $x$ ray distribution as well as the number and alignment of the detectors. Likewise, software analysis differs between manufacturers. Tylavsky and coworkers ${ }^{12}$ recently examined the use of fan beam DXA for the accurate assessment of body composition compared with a four compartment model (4C) as the criterion method. They found that the Hologic QDR 4500A systematically gave higher estimates of FFM and lower values for FM in older

Abbreviations: \%BF, percentage body fat; $\mathrm{BFLM}$, bone free lean mass; $B M C$, bone mineral content; $B M D$, bone mineral density; DXA, dual energy $x$ ray absorptiometry; FFM, fat free mass; FM, fat mass; HW, hydrostatic weighing; TBW, total body water 
adults than the 4C model. From their results they developed a correction factor to use with the Hologic fan beam instrument for assessing older adults. Similar research comparing fan beam DXA with standard laboratory reference methods has not been carried out. Therefore the purpose of this study was to compare body composition assessment using fan beam DXA (Lunar Prodigy) with body composition measurements from two and three compartment (2C and 3C) models.

\section{METHODS}

\section{Subjects}

Twenty five healthy men and 31 healthy, non-pregnant, premenopausal women aged 18-41 were enrolled. Before participation, written informed consent was obtained from each in accordance with the Human Subjects Review Committee and the Human Radiation Use Committee at the University of California, Davis.

\section{Protocol}

All measurements on each subject were made during one four hour period in the Human Performance Laboratory at the University of California, Davis. All subjects abstained from food for at least two hours before testing. Subjects were instructed to arrive hydrated and were not allowed to eat or drink throughout the protocol. They were introduced to all laboratory procedures before participation. In addition, women provided a urine specimen for pregnancy testing to confirm their eligibility for DXA scanning. Body density was obtained by HW, and the Siri two compartment equation $(2 \mathrm{C})^{13}$ was used to estimate $\% \mathrm{BF}$. Total body bone mineral was obtained using a dual energy $x$ ray absorptiometer and was incorporated into the $3 \mathrm{C}$ model. Height was obtained from a wall mounted stadiometer and recorded to the nearest $0.1 \mathrm{~cm}$. Weight was obtained from an electronic scale (model 58-501; Circuits \& Systems, East Rockaway, New York, USA) and recorded to the nearest $0.1 \mathrm{~kg}$.

\section{Hydrostatic weighing}

Whole body density was determined by HW using the highest underwater weight from three to six trials. ${ }^{14}{ }^{15}$ Underwater weight was determined to the nearest $0.1 \mathrm{~kg}$ on a $9 \mathrm{~kg}$ Chatillon scale. Weight in air was measured to the nearest $0.1 \mathrm{~kg}$ on an electronic scale (model 58-501; Circuits \& Systems). Residual volume was determined using a modification of Wilmore' $\mathrm{s}^{16}$ oxygen rebreathing technique and a 9 litre spirometer (Collins, Braintree, Massachusetts, USA), with a Perkin-Elmer Medical Gas Analyzer (model 1200; Norwalk, Connecticut, USA) to measure percentage expired nitrogen.

Reliability of the investigator using the HW method was shown by measuring three men and three women on three separate days. The mean \%BF for the six subjects was $20.88 \%$ for day $1,20.87 \%$ for day 2 , and $20.91 \%$ for day 3. Repeated measures analysis of variance indicated a significant difference between subjects, as expected, but no significant difference between days of testing. The coefficients of variation were $2.1 \%, 2.1 \%$, and $2.2 \%$ for day 1 , day 2 , and day 3 respectively (table 1 ).

\section{DXA}

Total body BMC (g), BFLM, FM, and \%BF were determined using a fan beam dual energy $x$ ray absorptiometer (Prodigy model; GE/Lunar Corp, Madison, Wisconsin, USA). FFM from DXA $\left(\mathrm{FFM}_{\mathrm{DXA}}\right)$ was calculated as the sum of BFLM and BMC. This newer DXA instrument uses a fan beam array rather than a pencil beam to distribute the $x$ rays. An array detector composed of cadmium/zinc/telluride elements was used to directly convert the $x$ rays into a digital signal. The Prodigy model uses a narrow $4.5^{\circ}$ angle fan beam oriented
Table 1 Within subject and between day reliability of body density determined by hydrostatic weighing

\begin{tabular}{llll}
\hline Subject & Day 1 & Day 2 & Day 3 \\
\hline 1 & 1.074 & 1.074 & 1.075 \\
2 & 1.047 & 1.048 & 1.046 \\
3 & 1.082 & 1.082 & 1.083 \\
4 & 1.036 & 1.035 & 1.035 \\
5 & 1.044 & 1.048 & 1.046 \\
6 & 1.026 & 1.024 & 1.024 \\
Mean & 1.0516 & 1.0517 & 1.0516 \\
SD & 0.022 & 0.023 & 0.022 \\
CV (\%) & 2.1 & 2.1 & 2.2 \\
\hline \multicolumn{4}{l}{ There were no significant differences between body density } \\
measured on different days. \\
CV, Coefficient of variation.
\end{tabular}

parallel to the longitudinal axis of the subject and scans in a transverse pattern across the body. Each fan beam pass overlaps slightly with the previous pass creating multiple images. By sliding these overlapping images together for a match (multiple view image reconstruction), the true distance of the object from the detector can be determined, thus reducing magnification effects on the determination of $\mathrm{BMC}$, bone area, and soft tissue. The amount of absorbed energy from the $x$ ray source is directly proportional to BMC and $\mathrm{BMD}$, which is measured with a precision better than $1 \%{ }^{1}$ BMD is expressed as an areal density in $\mathrm{g} / \mathrm{cm}^{2}$ and represents the bone mass per unit of projected bone area. Each scan was completed according to the manufacturer's instructions.

Subjects were instructed to wear metal free clothing and to remove all jewelry and other metal before the scan. If a subject reported to the laboratory wearing clothing that contained metal-for example, zippers, snaps, etc-then he/ she changed into cotton hospital scrubs for the DXA measurement.

Reliability of the DXA was assessed by phantom scans throughout the duration of the study. Internal laboratory calibration of this instrument has shown its reliability with a coefficient of variation of $0.075 \%$ for BMD. The coefficient of variation for the estimate of FM was $0.6 \%$. All scans were performed by one of two licensed DXA technicians. Between operator reliability was determined by repeated testing of 10 volunteers. Each operator tested each volunteer twice within the same week. A two tailed $t$ test indicated no significant differences between operators for either bone values or body composition values.

\section{Multicompartment equation}

To correct for assumptions based on the reference body when using HW, we used a 3C model based on the work of Lohman ${ }^{17}$ that corrected for differences in BMC $\left(3 \mathrm{C}_{\mathrm{BMC}}\right)$. Total body mineral content was calculated by multiplying the BMC by 1.279 to adjust for the non-osseous mineral content of the body. Lohman's equation is as follows, where M is total mineral content as a fraction of body weight:

$\%_{\mathrm{BF}_{3 \mathrm{CBMC}}}=\left(\left(6.386 / \mathrm{D}_{\mathrm{b}}\right)+3.96 \mathrm{M}-6.09\right) \times 100$

\section{Statistical analysis}

Descriptive statistics were used to characterise the subjects, and repeated measures analysis of variance was used to determine investigator reliability for HW. Pearson productmoment correlation coefficients were examined for all pairwise comparisons between \%BF values from each method $\left(2 \mathrm{C}, 3 \mathrm{C}_{\mathrm{BMC}}\right.$, and DXA). Bland-Altman plots $^{18}$ were used to examine the results for bias between the methods. Analysis of variance was used to test for significant differences among 
Table 2 Physical characteristics of participants

\begin{tabular}{|c|c|c|c|}
\hline & $\begin{array}{l}\text { Total group } \\
(n=56)\end{array}$ & $\begin{array}{l}\text { Men } \\
(n=25)\end{array}$ & $\begin{array}{l}\text { Women } \\
(n=31)\end{array}$ \\
\hline Age (years) & $25.7(5.1)$ & $26.2(3.9)$ & $25.4(6.0)$ \\
\hline Weight $(\mathrm{kg})$ & 70.7 (14.4) & $82.8(11.7)^{*}$ & 60.9 (7.3) \\
\hline Height $(\mathrm{cm})$ & $173.4(10.0)$ & $181.0(7.3)^{*}$ & 167.3 (7.3) \\
\hline $\mathrm{FFM}_{2 \mathrm{C}}$ & $57(13.5)$ & $69.8(8.3)^{*}$ & $46.6(5.4)$ \\
\hline $\mathrm{FFM}_{3 \mathrm{CBMC}}$ & 57.4 (14.4) & $71.1(9.1)^{*}$ & $46.4(5.4)$ \\
\hline FFM & 55.4 (13.9) & $68.6(8.7)^{*}$ & $44.7(5.8)$ \\
\hline$\% \mathrm{BF}_{2 \mathrm{C}}$ & 19.7 (7.7) & $15.3(6.9)^{*}$ & $23.3(6.4)$ \\
\hline$\% \mathrm{BF}_{3 С \mathrm{CMC}}$ & $19.2(8.6)$ & $13.7(7.0)^{*}$ & 23.6 (7.2) \\
\hline$\% \mathrm{BF}_{\mathrm{DXA}}$ & 21.7 (8.4) & $16.6(6.8)^{*}$ & $25.8(7.2)$ \\
\hline TBBMC $(g)$ & $3092(643)$ & 3603 (484) & 2648 (380) \\
\hline
\end{tabular}

FFM, Fat free mass; \%BF percentage body fat; $2 \mathrm{C}$, determinations performed by hydrostatic weighing using a two compartment model; 3CBMC, a two compartment model adjusted for variation in bone mineral content; DXA, values were obtained from fan beam dual energy $x$ ray absorptiometer; TBBMC, total body bone mineral content. *Significantly different from women $(p<0.05)$.

the methods and between sexes for the estimation of \%BF. The Statistical Analysis Software (SAS, version 8) was used for analysis of variance results. Significance was set at the 0.05 level of probability.

\section{RESULTS}

The mean age, height, and weight for the 56 subjects was 25.7 years, $173.4 \mathrm{~cm}$, and $70.7 \mathrm{~kg}$ respectively. The mean age, height, and weight for the men $(n=25)$ and women are shown separately in table 2 with other physical characteristics. The men were significantly taller and heavier than the women and had more FFM and less body fat.

Analysis of variance indicated that, for the whole group, $\% \mathrm{BF}$ results obtained by the $2 \mathrm{C}$ and $3 \mathrm{C}_{\mathrm{BMC}}$ models and DXA were not different from each other (table 2). There was, however, a significant difference in \%BF between the sexes for all methods-for example, men were less fat than women for all methods. The lack of a significant difference in \%BF among these methods is also reflected in the estimates of FFM, where no differences were observed in the total group for $\mathrm{FFM}_{2 \mathrm{C}}, \mathrm{FFM}_{3 \mathrm{CBMC}}$, and $\mathrm{FFM}_{\mathrm{DXA}}$. Regression analysis showed a range of correlations between the methods from 0.919 to 0.953 . Figures $1-3$ show comparisons of methods.

Bland-Altman plots $^{18}$ showed a trend for DXA measurements to overestimate \%BF compared with the $2 \mathrm{C}$ (fig 4 ) and $3 \mathrm{C}_{\mathrm{BMC}}$ (fig 5) models. Comparing \%BF $\mathrm{BXA}_{\mathrm{DA}}$ with the mean of the $\% \mathrm{BF}_{2 \mathrm{C}}$ and $\% \mathrm{BF}_{\mathrm{DXA}}$ methods resulted in 42 data points out of 56 above the mean of the two methods with a mean difference of $1.0 \%$ and a standard deviation of $1.2 \%$.

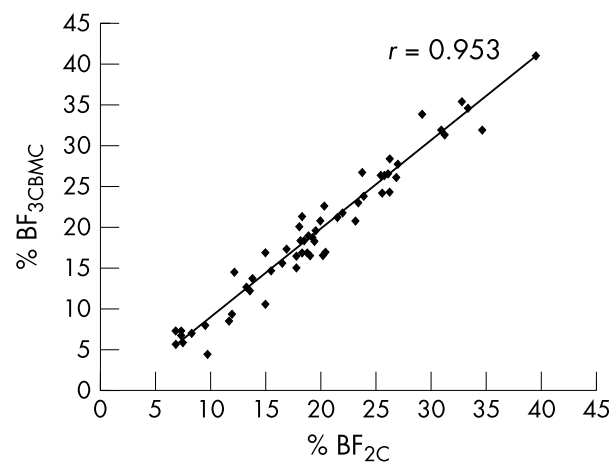

Figure 1 Comparison of $\% \mathrm{BF}_{3 \mathrm{CBMC}}$ with $\% \mathrm{BF}_{2 \mathrm{C}}$ in young adult men and women where \% $\mathrm{BF}$ is percentage body fat, $2 \mathrm{C}$ indicates determination performed by hydrostatic weighing using a two compartment model, and $3 C B M C$ is a two compartment model adjusted for variation in bone mineral content.

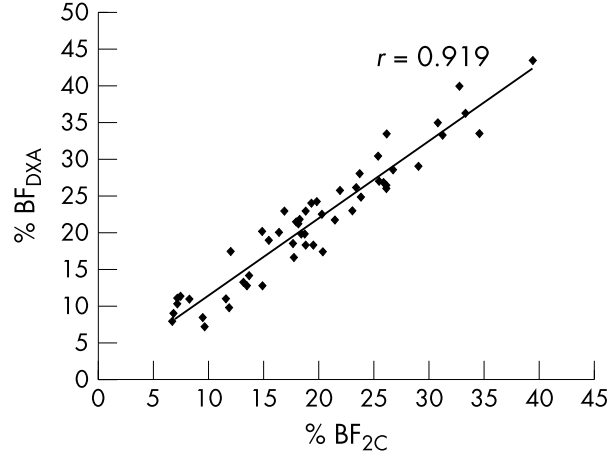

Figure 2 Comparison of $\% \mathrm{BF}_{\mathrm{DXA}}$ with $\% \mathrm{BF}_{2 \mathrm{C}}$ in young adult men and women where $\% \mathrm{BF}$ is percentage body fat, $2 \mathrm{C}$ indicates determination performed by hydrostatic weighing using a two compartment model, and DXA indicates determination using dual energy $x$ ray absorptiometry.

Similarly, a comparison of $\% \mathrm{BF}_{\mathrm{DXA}}$ with the mean of the $\% \mathrm{BF}_{\mathrm{DXA}}$ and $\% \mathrm{BF}_{3 \mathrm{CBMC}}$ methods showed that 53 out of 56 data points were above the mean of the two methods. This difference averaged $1.45 \%$ with a standard deviation of $0.86 \%$.

\section{DISCUSSION}

In this study, we compared \%BF determinations from a fan beam DXA instrument with those from $2 \mathrm{C}$ and $3 \mathrm{C}_{\mathrm{BMC}}$. Analysis of variance indicated no significant difference in \%BF determined by the three methods. Bland-Altman plots, used to evaluate potential bias in the methods, showed that $\% \mathrm{BF}$ values determined by fan beam DXA were only slightly higher than those determined by either the $2 \mathrm{C}$ or $3 \mathrm{C}_{\mathrm{BMC}}$ models: by $0.68-1.45 \%$. The findings of the comparison of $\% \mathrm{BF}_{\mathrm{DXA}}$ with $\% \mathrm{BF}_{2 \mathrm{C}}$ and $\% \mathrm{BF}_{3 \mathrm{BMC}}$ were as expected. This sample of participants consisted of healthy young adult men and premenopausal women for whom the assumptions on the constancy of body compartments should be reasonably accurate. Therefore we expected to find no differences among the methods. Furthermore, it is our belief that the comparison of $\% \mathrm{BF}_{\mathrm{DXA}}$ with the $\% \mathrm{BF}_{2 \mathrm{C}}$ and $\% \mathrm{BF}_{3 \mathrm{CBMC}}$ models is appropriate and accurately reflects the performance of the fan beam DXA. Our results are the first reported for Lunar Prodigy fan beam DXA and therefore there are no other direct comparisons for discussion.

Other research, however, has evaluated the fan beam DXA method, ${ }^{12}{ }^{19}$ specifically using the Hologic QDR 4500A. The first of these studies compared the QDR 4500A with a 4C model for assessment of FFM in older men and women

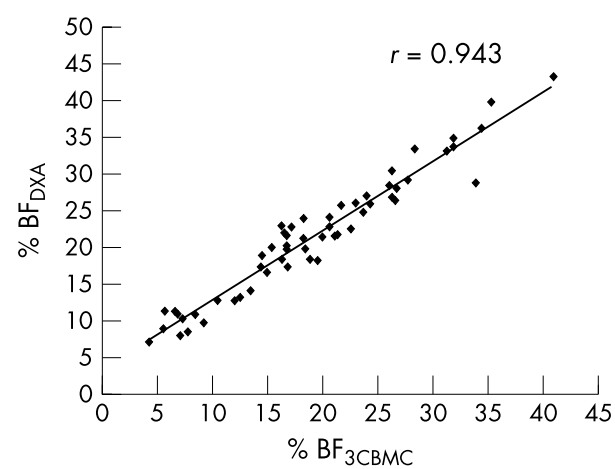

Figure 3 Comparison of $\% \mathrm{BF}_{\mathrm{DXA}}$ with $\% \mathrm{BF}_{3 \mathrm{CBMC}}$ in young adult men and women where \% $\mathrm{BF}$ is percentage body fat, $3 \mathrm{CBMC}$ is a two compartment model adjusted for variation in bone mineral content, and DXA indicates determination using dual energy $x$ ray absorptiometry. 


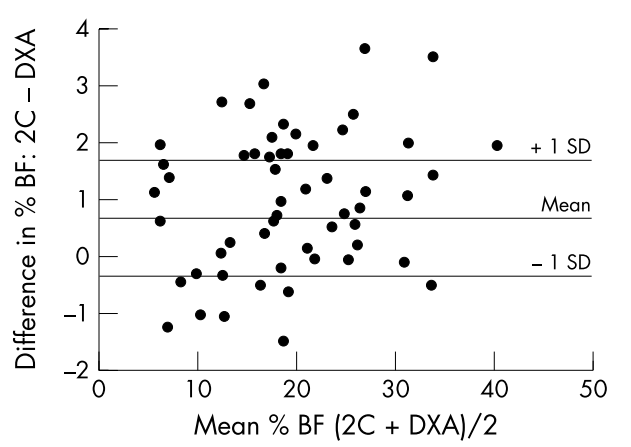

Figure 4 Bland-Altman plot comparing the difference between $\% \mathrm{BF}_{2 \mathrm{C}}$ and $\% \mathrm{BF}_{\mathrm{DXA}}$ with the mean of $\% \mathrm{BF}_{2}$ and $\% \mathrm{BF}_{\mathrm{DXA}}$ where $\% \mathrm{BF}$ is percentage body fat, $2 \mathrm{C}$ indicates determination performed by hydrostatic weighing using a two compartment model, and DXA indicates determination using dual energy $x$ ray absorptiometry.

(70-79 years of age). The QDR 4500A overestimated FFM and underestimated FM. These overestimations and underestimations, however, were systematic and thus a correction factor was developed. Tylavsky et al ${ }^{19}$ compared the QDR 4500A fan beam DXA with an estimate of FFM obtained from total body water (TBW) measurements with changes in body weight. Results from the fan beam DXA were corrected using the correction factor previously developed..$^{12}$ The corrected FFM was then compared with FFM estimated from TBW. Overall, the fan beam method provided higher estimates of the change in soft tissue lean mass and lower estimates of the change in FM. The correlations between the change in soft tissue lean mass and the change in FFM from TBW was 0.61. However, the regression coefficients for the slope and intercept included 1 and 0 respectively. Bland-Altman analysis indicated that the fan beam system provided higher estimates of change in soft tissue lean mass and lower estimates of loss of FM than the TBW method. However, the authors noted that significant decreases in bone area (which can affect BMD values) were not observed with weight change, as previously observed with pencil beam instruments. ${ }^{20}$ Finally, the authors compared the results from the fan beam DXA with those obtained with a pencil beam DXA and concluded that the results were not interchangeable between machines.

Under other circumstances-for example, postmenopausal women, disease conditions, disabilities-in which body compartments may not remain constant, we do not know if fan beam DXA would be accurate. However, this study supports Lunar Prodigy fan beam DXA as an acceptable

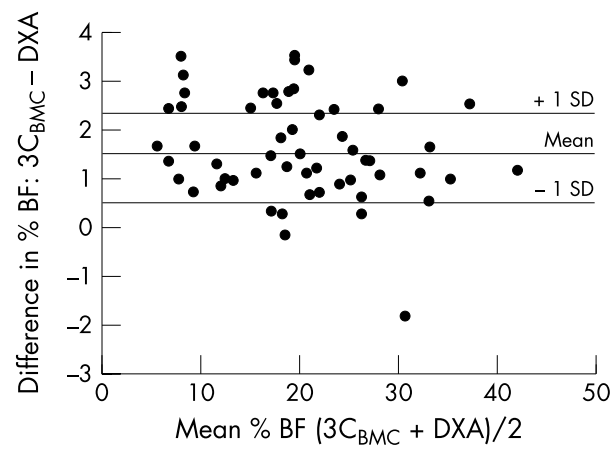

Figure 5 Bland-Altman plot comparing the difference between $\% \mathrm{BF}_{3 C B M C}$ and $\% \mathrm{BF}_{\mathrm{DXA}}$ with the mean of $\% \mathrm{BF}_{3 C B M C}$ and $\% \mathrm{BF}_{\mathrm{DXA}}$ where $\% \mathrm{BF}$ is percentage body fat, $3 \mathrm{CBMC}$ is a two compartment model adjusted for variation in bone mineral content, and DXA indicates determination using dual energy $x$ ray absorptiometry.
Take home message

Fan beam DXA for assessment of body composition, while promising, has not been fully studied. This research suggests that the Lunar instrument is accurate with young adults, but other research indicates a bias with the Hologic instrument when used with older subjects. Fan beam DXA for body composition assessment needs further study before a recommendation on its accuracy can be made.

method for body composition assessment in adults aged 1845. Although a reference 4C model with TBW derived from $\mathrm{D}_{2} \mathrm{O}$ dilution was not available in this study, HW has long been considered the optimum method of measuring body composition for young adult men and women. Our findings show that $\% \mathrm{BF}_{\mathrm{DXA}}$ values from a fan beam instrument were not different from estimates obtained by HW in this group of adults. In addition, when $\% \mathrm{BF}_{\mathrm{DXA}}$ was compared with a $3 \mathrm{C}$ model adjusted for variation in BMC, no significant differences were observed.

DXA is also beneficial for several other reasons not directly related to accuracy or reliability. Because it can measure BMC and soft tissue composition in one examination, it is superior to all the other methods. It provides two clinical measurements and does it in less time and with less physical and psychological stress. Generally accepted methods such as skinfolds, HW, and Bod Pod all result in some physical discomfort for the subject. DXA has none of these discomforts. Finally, DXA is a convenient technique for body composition analysis. It costs approximately the same as other instrumentation needed to perform the multicompartment methods and takes up no more space than other standard equipment for $2 \mathrm{C}$ and $3 \mathrm{C}$ analysis. In addition, DXA analysis provides data not available from the other methods, namely BMD, BMC, and regional body composition. The fan beam DXA instrument is considerably faster than previous models, requires less subject participation than other reference techniques, and may be more useful in the assessment of special populations in which HW is difficult to execute. Clearly, there are numerous reasons to further validate this technology and make adjustments as necessary.

\section{CONCLUSION}

There are many potential benefits of using DXA for body composition assessment. Other studies have also shown its reliability and validity. ${ }^{45}$ This study further shows the validity and accuracy of the Lunar Prodigy fan beam DXA instrument compared with $2 \mathrm{C}$ and $3 \mathrm{C}_{\mathrm{BMC}}$ models. Future research is needed, however, to confirm the validity and accuracy of the fan beam DXA compared with a 4C model. Also, the validity and accuracy of this particular fan beam DXA as a technique for all populations and investigative conditions has not been fully investigated and warrants further study. Finally, results from this study suggest that future research efforts with fan beam DXA will be promising.

\section{Authors' affiliations \\ J Norcross, University of California, Davis, CA, USA \\ M D Van Loan, USDA-Western Human Nutrition Research Center, University of California, Davis}

\section{REFERENCES}

1 Cullum ID, Ell PJ, Ryder JP. X-ray dual-photon absorptiometry: a new method for the measurement of bone density. Br J Radiol 1989:62:587-92.

2 Gotfredson AJ, Jensen J, Christiansen B, et al. Measurement of lean body mass and total body fat using dual photon absorptiometry. Metabolism 1986;35:88-93. 
3 Heymsfield SB, Wang Z, Wang J, et al. Theoretical foundation of dual energy $x$-ray absorptiometry (DEXA) soft tissue estimates: validation in situ and in vivo. FASEB J 1994;8A:278

4 Mazess RB, Barden HS, Bisek JP, et al. Dual-energy x-ray absorptiometry for total-body and regional bone-mineral and soft-tissue composition. Am J Clin Nutr 1990;51:1106-12.

5 Van Loan MD, Mayclin PL. Body composition assessment: dual-energy x-ray absorptiometry (DEXA) compared to reference methods. Eur J Clin Nutr 1992:46:125-30

6 Laskey MA, Lyttle KD, Flarman ME, et al. The influence of tissue depth and composition on the performance of the Luan dual energy $x$-ray absorptiometer whole body scanning mode. Eur J Clin Nutr 1992;46:39-45.

7 Jebb SA, Goldberg GR, Marinos E. DXA measurements of fat and bone mineral density in relation to depth and adiposity. In: Ellis KJ, Eastmen JD, eds. Human body composition. New York: Plenum, 1993:115-19.

8 Tothill PA, Avenell J, Reid L, et al. Comparisons between Hologic, Lunar and Norland dual-energy $\mathrm{x}$-ray absorptiometers and other techniques used for whole-body soft tissue measurements. Eur J Clin Nutr 1994;48:781-94.

9 Van Loan MD, Keim NL, Berg K, et al. Evaluation of body composition by dual energy $x$-ray absorptiometry and two different software packages. Med Sci Sports Exerc 1995;27:587-91.

10 Kohrt WM. Body composition by DXA: tried and true? Med Sci Sports Exerc 1995;27:1349-53.

11 Roubenoff R, Kehayias JJ, Dawson-Hughes B, et al. Use of dual-energy x-ray absorptiometry in body composition studies: not yet a "gold standard." Am J Clin Nutr 1993;58:589-91.
12 Tylavsky F, Lohman T, Blunt BA, et al. QDR4500A DXA overestimates fat-free mass compared with criterion methods. J Apply Physiol 2003;94:959-65

13 Siri WE. Body composition from fluid spaces and density: analysis of methods. In: Tobias CA, Lawrence JH, eds. Advances in biological and medical physics. New York: Academic, 1956;4:239-80.

14 Bonge $D$, Donnelly JE. Trials to criteria for hydrostatic weighing at residual volume. Res Q Exerc Sport 1989;60:176-9.

15 Goldman RF, Buskirk ER. Body volume measurement by underwater weighing: description of a method. In: Brozek J, Henschel A, eds. Techniques for measuring body composition. Washington DC: NASNRC, 1961:77-89.

16 Wilmore J. A simplified method for determining residual lung volumes. J Appl Physiol 1969;27:96-100.

17 Lohman TG. Applicability of body composition techniques and constants for children and youths. Exerc Sports Sci Rev 1986;14:325-57.

18 Bland JM, Altman DG. Statistical methods for assessing agreement between two methods of clinical measurement. Lancet 1986;1 307-10

19 Tylavsky FA, Lohman TG, Dockrell M, et al. Comparison of the effectiveness of 2 dual-energy $x$-ray absorptiometers with that of total body water and computed tomography in assessing changes in body composition during weight change. Am J Clin Nutr 2003;77:356-63.

20 Van Loan MD, Johnson HL, Barbieri TF. Effect of weight loss on bone mineral content and bone mineral density in obese women. Am J Clin Nutr 1998;67:734-8. 